OPEN ACCESS

Edited by:

Milica S. Prostran,

University of Belgrade, Serbia

Reviewed by:

Camillo Marra,

Institute of Neurology, Catholic University of the Sacred Heart,

Rome, Italy

Boon-Seng Wong,

Singapore Institute of Technology,

Singapore

*Correspondence:

Guanjun Li

liguanjun66@126.com

Shifu Xiao

xiaoshifu@msn.com

†These authors have contributed equally to this work

Received: 22 February 2019 Accepted: 03 July 2019 Published: 30 July 2019

Citation:

Li W, Sun L, Li G and Xiao S (2019) Prevalence, Influence Factors and Cognitive Characteristics of Mild Cognitive Impairment in Type 2 Diabetes Mellitus. Front. Aging Neurosci. 11:180 doi: 10.3389/fnagi.2019.00180

\section{Prevalence, Influence Factors and Cognitive Characteristics of Mild Cognitive Impairment in Type 2 Diabetes Mellitus}

\author{
Wei $\mathrm{Li}^{1 \dagger}$, Lin Sun ${ }^{1 \dagger}$, Guanjun $\mathrm{Li}^{1 *}$ and Shifu Xiao ${ }^{1 *}$ \\ ${ }^{1}$ Department of Geriatric Psychiatry, Shanghai Mental Health Center, Shanghai Jiao Tong University School of Medicine, \\ Shanghai, China, ${ }^{2}$ Alzheimer's Disease and Related Disorders Center, Shanghai Jiao Tong University, Shanghai, China
}

Background: Type 2 diabetes mellitus (T2DM) is considered as an independent risk factor for mild cognitive impairment (MCl). This study was performed to investigate the prevalence, influencing factors and cognitive characteristics of $\mathrm{MCl}$ in elderly patients with T2DM in China.

Methods: In the cross-sectional study, we performed cluster random sampling of 3,246 people age 60 years and older across the country. All participants were interviewed and screened for T2DM and $\mathrm{MCl}$. A total of 341 subjects were diagnosed of $\mathrm{MCl}$ according to the criteria of Petersen, and a total of 256 subjects were diagnosed of T2DM by using the American Diabetes Association criteria Among the 256 T2DM people, 56 were also diagnosed with $\mathrm{MCl}$. Logistic regression analyses were performed to evaluate risk and protective factor for $\mathrm{MCl}$ with T2DM. We also assessed their cognitive function by using the Mini-mental State Examination (MMSE), Montreal Cognitive Assessment (MoCA), Digit span, Associative Learning Test (ALT), Visual Identification Test (VIT), Verbal Fluency (VF), Wechsler Adult Intelligence Scale (WAIS)-III Block Design, WAIS-III picture completion and Auditory Verbal Learning Test (AVLT).

Results: Among the 256 T2DM patients, 56 were diagnosed with $\mathrm{MCl}$, and the prevalence of $\mathrm{MCl}$ in T2DM was 21.8\%. Multivariate logistic regression analyses showed that depression ( $p=0.002$, OR $=6.220,95 \% \mathrm{Cl}: 2.005-19.290$ ) was a risk factor for $\mathrm{MCl}$ among T2DM patients, while education ( $p<0.001$, OR $=0.869,95 \% \mathrm{Cl}: 0.805-0.983$ ) was a protective one. All the scores of neuropsychological tests (except for MMSE) in T2DM patients with $\mathrm{MCl}$ were lower than those without $\mathrm{MCl}(p<0.05)$, but there was no statistical difference $(p>0.05)$ in neuropsychological tests between T2DM-MCl group and No-T2DM-MCl group. Linear regression analysis showed that the drug treatment of diabetes was positively correlated ( $t=2.263, p=0.025$ ) with the total score of auditory word tests.

Conclusions: The present study suggests a high prevalence of $\mathrm{MCl}$ among Chinese T2DM patients. Depression is a risk factor for $\mathrm{MCl}$, while education is a protective one. T2DM patients with $\mathrm{MCl}$ often show comprehensive cognitive impairment, and the drug treatment of diabetes might help to improve cognitive function.

Keywords: T2DM, MCI, Chinese elderly, auditory verbal learning test, cross-section study 


\section{INTRODUCTION}

Type 2 diabetes mellitus (T2DM) is a common metabolic disorder characterized by high blood sugar, insulin resistance, and a relative lack of insulin (Yang et al., 2018). A recent meta-analysis (Yang et al., 2016) pointed out that the overall prevalence $(9.1 \%)$ of T2DM in China has been increasing since the 1970s, and it increased rapidly with age. In addition to the well-known connection between T2DM and peripheral nervous system disease, diabetes may also cause damage to the central nervous system. What's more, patients with T2DM generally show worse cognitive performance (Kodl and Seaquist, 2008) and a higher risk of dementia (Arvanitakis et al., 2004; Smith et al., 2010).

Mild cognitive impairment (MCI) is a transitional and possibly modifiable stage between normal cognitive aging and dementia (Petersen, 2011). Compared to healthy individual, people with MCI often have an increased risk to develop dementia (Petersen et al., 1999). Previous studies suggest that T2DM is not only a risk factor for MCI, but also promotes the transformation of MCI into dementia (Cukierman et al., 2005; Li et al., 2016). Therefore, early treatment of T2DM in patients with MCI is critical for improving their prognosis (Cholerton et al., 2016; Xu et al., 2016).

China's population accounts for $21 \%$ of the world population and $1 / 3$ of the Asian population. However, few studies have examined the prevalence of MCI in Chinese T2DM patients [we only found one such study (Gao et al., 2016), but their conclusions are not representative of the country]. So we conduct this national study to explore the prevalence, influence factors and cognitive characteristics of $\mathrm{MCI}$ in T2DM among Chinese community elderly.

\section{MATERIALS AND METHODS}

\section{Study Population}

The China Longitudinal Aging Study (CLAS) is a populationbased cohort that is studying randomly sampled participants living in the city communities in China. For a detailed description of the study design, see Xiao et al. (2013). Briefly, 3,246 participants aged 60 years or more from all over the country underwent a baseline examination including a review of their medical history, physical and neurological examinations, laboratory tests, and magnetic resonance imaging (MRI) scans. The recruitment procedure yielded an almost representative sample for the respective communities. The following data, such as name, gender, age, education and daily living information (hobby, dietary preferences, sleeping patterns, smoking history, consumption of tea and alcohol and physical activities) were also collected by standardized questionnaire.

Of the original 3,246 elderly subjects, 389 participants were excluded for the incomplete data; 1,331 people were excluded because they did not meet the diagnostic criteria for MCI or normal cognitive elderly; Among those MCI patients $(n=341)$ and normal people $(n=1,185)$, there were also 256 people

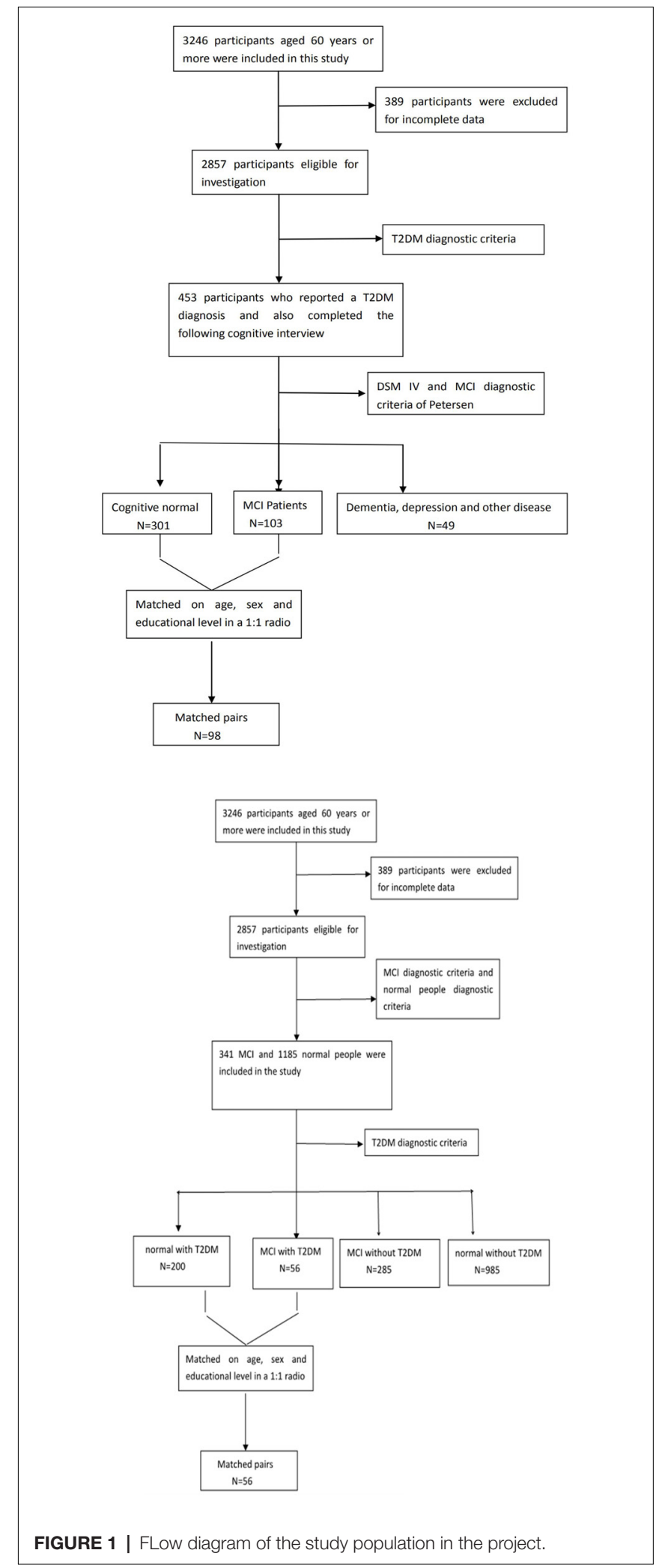

(T2DM-MCI, $n=56$; T2DM-normal, $n=200$ ) were diagnosed with diabetes. Figure 1 presents an overview of the study design and sampling procedures. 
TABLE 1 | Demographic, health, and diabetes-related for three groups.

\begin{tabular}{|c|c|c|c|c|c|}
\hline Characteristics & T2DM-MCI $(n=56)$ & No-T2DM-MCI $(n=285)$ & T2DM-Normal $(n=200)$ & $\boldsymbol{F}$ & $P$-value \\
\hline Age, years & $74.23 \pm 6.93$ & $75.90 \pm 7.57$ & $71.12 \pm 7.51$ & 23.997 & $<0.001^{*}$ \\
\hline Education, years & $4.73 \pm 4.94$ & $4.99 \pm 4.79$ & $9.00 \pm 4.90$ & 42.014 & $<0.001^{*}$ \\
\hline $\mathrm{BMI}, \mathrm{Kg} / \mathrm{m}^{2}$ & $24.38 \pm 2.91$ & $23.51 \pm 4.11$ & $24.17 \pm 3.20$ & 1.698 & 0.185 \\
\hline SBP, mmHg & $134.91 \pm 17.27$ & $129.05 \pm 14.73$ & $129.77 \pm 15.03$ & 3.537 & $0.030^{*}$ \\
\hline DBP, mmHg & $75.12 \pm 13.40$ & $76.65 \pm 8.12$ & $77.57 \pm 8.58$ & 1.758 & 0.173 \\
\hline Duration of T2DM, years & $8.89 \pm 7.19$ & 0 & $8.86 \pm 7.35$ & 1.156 & 0.317 \\
\hline Fasting blood-glucose, $\mathrm{mmol} / \mathrm{L}$ & $12.78 \pm 5.35$ & $6.87 \pm 4.374$ & $12.09 \pm 5.49$ & 0.355 & 0.701 \\
\hline Tri-glyceride, $\mathrm{mmol} / \mathrm{L}$ & $3.42 \pm 1.63$ & $6.96 \pm 4.38$ & $3.60 \pm 2.26$ & 2.767 & 0.090 \\
\hline Cholesterol, mmol/L & $4.07 \pm 4.91$ & $6.95 \pm 1.50$ & $7.25 \pm 2.21$ & 0.343 & 0.717 \\
\hline Male (\%) & 37.5 & 37.5 & 50.5 & 8.678 & $0.013^{*}$ \\
\hline Smoking (\%) & 24.1 & 26.3 & 25.0 & 0.609 & 0.738 \\
\hline Drinking alcohol (\%) & 14.3 & 18.9 & 19.0 & 0.731 & 0.694 \\
\hline Drinking tea (\%) & 35.7 & 36.5 & 51.0 & 11.114 & $0.004^{*}$ \\
\hline Eating fish (\%) & 85.7 & 86.7 & 93.0 & 5.427 & 0.066 \\
\hline Taking exercise (\%) & 69.6 & 55.4 & 75.0 & 20.374 & $<0.001^{\star}$ \\
\hline Hobby: reading (\%) & 10.7 & 11.9 & 26.5 & 19.367 & $<0.001^{*}$ \\
\hline Hobby: listening to music (\%) & 5.4 & 13.7 & 21.5 & 10.478 & $0.005^{*}$ \\
\hline Surfing the internet (\%) & 0 & 2.5 & 7.5 & 10.312 & $0.006^{*}$ \\
\hline Depression (\%) & 16.1 & 9.1 & 3.5 & 11.210 & $0.004^{*}$ \\
\hline Sleep disorder (\%) & 6.8 & 15.1 & 14.0 & 5.644 & 0.059 \\
\hline
\end{tabular}

${ }^{*} p<0.05$.

TABLE 2 | Risk and protective factors for MCl in T2DM patients.

\begin{tabular}{|c|c|c|c|c|c|c|c|c|}
\hline \multirow[t]{2}{*}{ Variables } & \multirow[t]{2}{*}{$B$} & \multirow[t]{2}{*}{ SE } & \multirow[t]{2}{*}{ Wals } & \multirow[t]{2}{*}{$d f$} & \multirow[t]{2}{*}{$p$} & \multirow[t]{2}{*}{ OR } & \multicolumn{2}{|c|}{ The $95 \% \mathrm{Cl}$ of OR } \\
\hline & & & & & & & Lower limit & Upper limit \\
\hline Education & -0.140 & 0.039 & 12.935 & 1 & 0.001 & 0.869 & 0.805 & 0.983 \\
\hline Depression & 1.828 & 0.577 & 10.017 & 1 & 0.002 & 6.220 & 2.005 & 19.290 \\
\hline
\end{tabular}

TABLE 3 | Results of global cognitive functioning tests and neuropsychological tests in different cognitive domains among three groups.

\begin{tabular}{|c|c|c|c|c|}
\hline Neuropsychological test & T2DM-MCI $(n=56)$ & No-T2DM-MCI $(n=56)$ & T2DM-Normal $(n=56)$ & $p$-value \\
\hline Age, years & $74.23 \pm 6.93$ & $74.18 \pm 6.76$ & $74.68 \pm 6.66$ & 0.912 \\
\hline Education, years & $4.73 \pm 4.94$ & $4.78 \pm 5.00$ & $4.59 \pm 4.03$ & 0.976 \\
\hline Male (\%) & 37.5 & 37.5 & 37.5 & 1.000 \\
\hline \multicolumn{5}{|l|}{ Diabetes related information } \\
\hline Duration of diabetes, years & $8.89 \pm 7.19$ & 0 & $7.53 \pm 6.90$ & 0.312 \\
\hline Fasting blood glucose, mmol/L & $12.78 \pm 5.35$ & 0 & $11.96 \pm 6.06$ & 0.491 \\
\hline Drug therapy (\%) & 98.2 & 0 & 85.5 & $0.016^{\star}$ \\
\hline \multicolumn{5}{|l|}{ Neuropsychological tests } \\
\hline MMSE & $22.13 \pm 5.39$ & $22.24 \pm 5.09$ & $24.32 \pm 4.47$ & $0.035^{\star}$ \\
\hline MoCA & $15.59 \pm 6.33$ & $15.91 \pm 6.34$ & $18.59 \pm 6.05$ & $0.022^{*}$ \\
\hline Digit Span (forward) & $5.87 \pm 2.95$ & $6.68 \pm 3.19$ & $7.83 \pm 2.77$ & $0.003^{*}$ \\
\hline Digit Span (backward) & $3.16 \pm 2.07$ & $3.43 \pm 2.01$ & $4.61 \pm 2.83$ & $0.001^{*}$ \\
\hline Auditory verbal learning test (T) & $41.45 \pm 16.96$ & $38.65 \pm 21.16$ & $54.89 \pm 23.53$ & $0.002^{*}$ \\
\hline Auditory verbal learning test 1 & $3.54 \pm 1.96$ & $3.65 \pm 2.38$ & $4.67 \pm 2.83$ & $0.029^{*}$ \\
\hline Auditory verbal learning test 2 & $5.41 \pm 2.46$ & $5.24 \pm 2.78$ & $6.56 \pm 3.03$ & $0.028^{*}$ \\
\hline Auditory verbal learning test 3 & $6.11 \pm 2.76$ & $5.92 \pm 3.23$ & $7.26 \pm 3.05$ & $0.048^{*}$ \\
\hline Auditory verbal learning test 4 & $6.42 \pm 2.90$ & $6.40 \pm 3.27$ & $7.98 \pm 3.00$ & $0.010^{*}$ \\
\hline Auditory verbal learning test 5 & $7.48 \pm 3.01$ & $6.88 \pm 3.40$ & $9.11 \pm 3.28$ & $0.010^{*}$ \\
\hline Auditory verbal learning test 6 & $4.48 \pm 3.48$ & $4.13 \pm 4.37$ & $6.13 \pm 3.76$ & $0.002^{*}$ \\
\hline Auditory verbal learning test 7 & $2.85 \pm 1.69$ & $3.17 \pm 1.78$ & $3.65 \pm 2.01$ & 0.079 \\
\hline Auditory verbal learning test 8 & $4.28 \pm 3.40$ & $3.73 \pm 3.88$ & $5.87 \pm 4.20$ & $0.013^{*}$ \\
\hline
\end{tabular}

${ }^{*} p<0.05$. 
TABLE 4 | Corrected the results of neuropsychological tests by using Bonferroni correction.

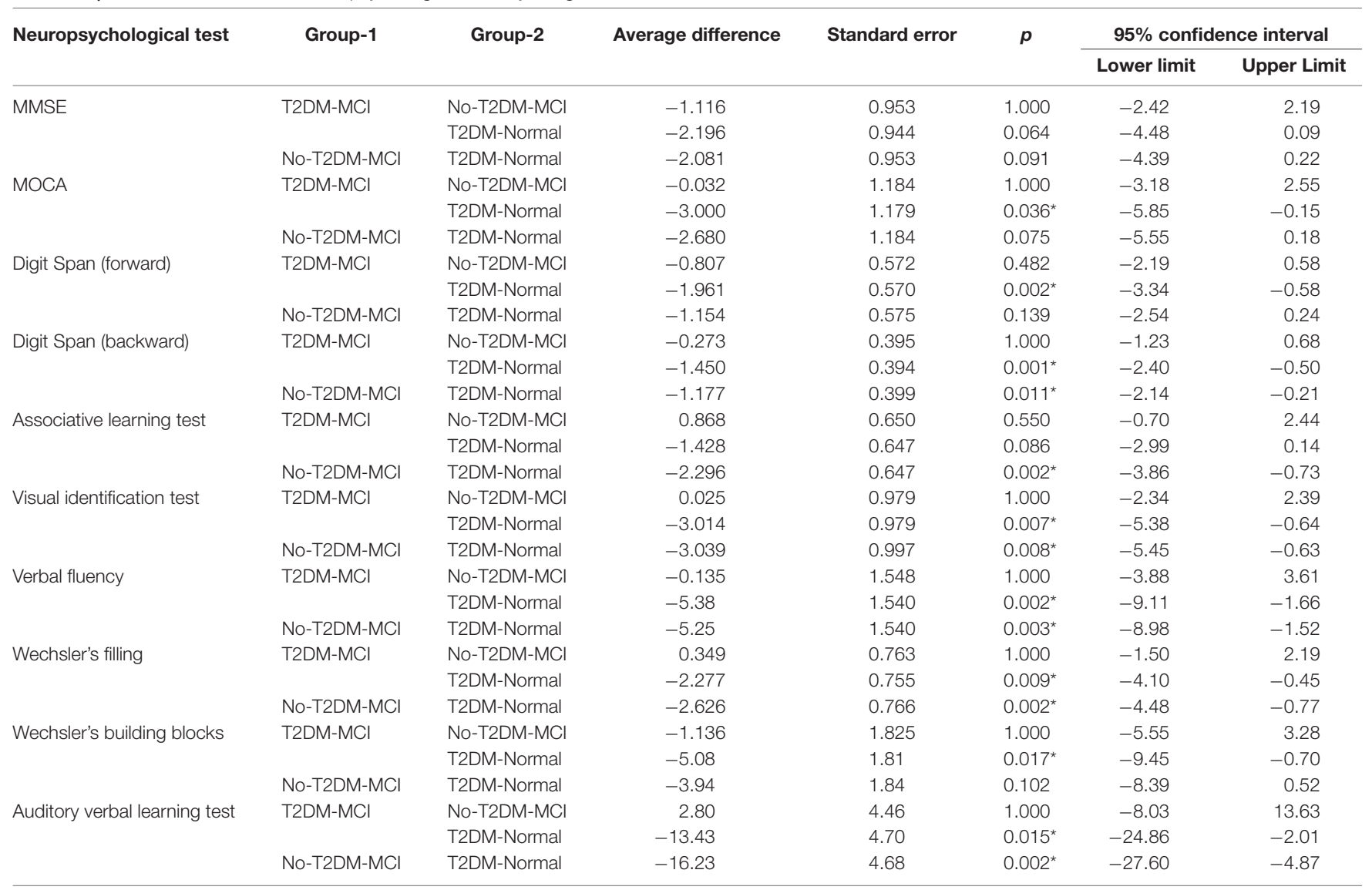

${ }^{*} p<0.05$.

$\overline{\text { TABLE } 5 \text { | Factors that might affect the score of the auditory vocabulary learning }}$ tests.

\begin{tabular}{lrrrrr}
\hline Group & $\boldsymbol{B}$ & $\begin{array}{c}\text { Standard } \\
\text { error }\end{array}$ & Standard & $\boldsymbol{t}$ & $\boldsymbol{p}$ \\
\hline Age & -1.096 & 0.268 & -0.349 & -4.093 & $<0.001$ \\
Education & 1.143 & 0.478 & 0.204 & 2.392 & 0.018 \\
Drug therapy (\%) & 14.412 & 6.396 & 0.178 & 2.263 & 0.025 \\
\hline
\end{tabular}

Ethical approval was obtained from Shanghai Mental Health Centre, and all the participants had signed an informed consent before the study was initiated.

\section{Measurement of T2DM}

Diabetic status was based on self-reported physician's diagnosis or treatment with insulin and/or oral hypoglycemic agents. Individuals were also considered to have T2DM if their fasting serum glucose level was $\geq 7.0 \mathrm{mmol} / \mathrm{L}(126 \mathrm{mg} / \mathrm{dl})$ or their 2 - $\mathrm{h}$ value in an oral glucose tolerance test was $\geq 11.1 \mathrm{mmol} / \mathrm{L}$ (200 mg/dl; Gao et al., 2015). Finally, 256 participants were considered to have T2DM.

\section{Diagnostic Criteria}

A diagnosis of MCI was based on the following criteria, which were adapted from the MCI diagnostic criteria of Petersen (Portet et al., 2006): (1) memory complaint, preferably corroborated by an informant; (2) objective memory impairment; (3) preservation of general cognitive function; (4) intact activities of daily living; and (5) absence of dementia. Finally, we found that there were 341 MCI patients in the whole population $(n=2,857)$, so the prevalence of MCI in the study population was $11.9 \%$. Of 256 T2DM subjects, 56 subjects were diagnosed with MCI, so the prevalence of MCI among T2DM patients was $21.8 \%$ Therefore, we concluded that the prevalence of MCI in diabetic patients was significantly higher than that in normal population (Pearson $\left.x^{2}=20.861, p<0.001\right)$.

\section{Cognitive Assessment}

Several psychological and psychosocial tests, including the Mini-Mental State Examination (MMSE; O'Bryant et al., 2008), the Montreal Cognitive Assessment (MoCA; Gil et al., 2015), Wechsler Adult Intelligence Scale (WAIS)-III Digit Span (Leung et al., 2011), Auditory Verbal Learning Test (AVLT; Hong et al., 2012), Associative Learning Test (ALT), Visual Identification Test (VIT), Verbal Fluency (VF), WAIS-III Block Design and WAIS-III picture completion were performed to evaluate the cognitive function, such as attention, semantic memory, visuospatial skills, psychomotor speed and executive function, of each subject. The Geriatric 
Depression Scale (Lin et al., 2016) was also used to exclude depression. All these tests were facilitated by an experienced neuropsychiatrist, and all the subjects were not informed of the study design.

\section{Statistical Analysis}

Statistical analysis was performed by using SPSS version 17.0. The continuous variable and categorical variables were presented as mean $\pm \mathrm{SD}$ and frequency (\%), respectively. All continuous data were tested for normally with the Kolmogorov-Smirnov test. Comparison of continuous data among groups was performed with the single factor analysis of variance (ANOVA) analysis and the results were corrected by using Bonferroni correction. The chi-square test was used for comparison of categorical data. Multiple logistic regression analysis was used for screening the risk and protective factors of MCI. A $P$-value $<0.05$ was considered to be significant.

\section{RESULTS}

Figure 1 shows the flow diagram of this study and the demographic variables were shown in Table 1. There were statistical differences $(p<0.05)$ in age, education, systolic blood pressure (SBP), gender, drinking tea, taking exercise, reading, listening to music, suffering the internet, and depression among the T2DM-MCI group, No-T2DM-MCI group and T2DM-Normal group. Then we brought these above variables into multiple regression equation, and the results showed that depression $(p=0.002$, OR $=6.220,95 \%$ CI: $2.005-19.290$ ) was a risk factor for the development of MCI in patients with diabetes, while education $(p<0.001, \mathrm{OR}=0.869,95 \% \mathrm{CI}$ : $0.805-0.983$ ) was an important protective factor (Table 2 shows the related results). However, We did not find a significant correlation ( $r=0.001, p=1.000)$ between MCI and T2DM through the correlation analysis, suggesting that T2DM might be a adjunctive risk factor for MCI. Next, we compared the cognitive characteristics among the three groups, and 56 pairs of age, sex and education-matched population were selected to participate in the final assessment. We found that there were statistical differences $(p<0.05)$ in the scores of MMSE, MoCA, Digit Span, ALT, VIT, VF, WAIS-III Block Design, WAIS-III picture completion and AVLTs among the three groups (Table 3). Then by using Bonferroni correction, we found that there was no statistical difference $(p>0.05)$ in neuropsychological tests between T2DM-MCI group and No-T2DM-MCI group. But there were statistical differences $(p<0.05)$ in the scores of MoCA, Digit Span, VIT, VF, WAIS-III Block Design, WAIS-III picture completion and AVLTs between T2DM-MCI group and T2DM-Normal group. And there were also statistical differences $(p<0.05)$ in the scores of Digit Span (backward), ALT, VIT, VF, WAIS-III picture completion, and AVLTs between No-T2DMMCI group and T2DM-Normal group (Table 4). Finally, by using linear regression analysis, we confirmed that the total scores of auditory learning tests were associated with age $(t=-4.093$, $p<0.001)$, education $(t=2.392, p=0.018)$ and treatment of diabetes with drugs $(t=2.263, p=0.025$; Table 5).

\section{DISCUSSION}

Our report presents the results of a national cross-sectional study that examined the prevalence, risk and protective factor as well as cognitive characteristics of MCI in a population of Chinese elderly patients with Type 2 diabetes. And this study revealed several interesting findings: (1) there was a very high prevalence of MCI among Chinese elderly with Type 2 diabetes; (2) depression was a risk factor for the development of MCI in patients with diabetes, while education was an important protective factor; (3) both overall and specific areas of cognitive function would decline in T2DM patients with MCI, but for patients with MCI, T2DM had no effect on cognitive function; and (4) promptly treating diabetes with drugs might improve the cognitive status of diabetics.

In this study, we found that the prevalence of MCI in Chinese elderly diabetic patients was $21.8 \%$, and it was lower than other data in the worldwide literature. For example, one population-based study suggested that the incidence of MCI in diabetic patients was around 28\% (Luchsinger et al., 2007), and another study reported the prevalence of MCI in elders was 31.5\% (Gorska-Ciebiada et al., 2014). However, a similar study conducted in China showed that the prevalence of MCI among the elderly population was only $13.5 \%$ (Gao et al., 2016). We suspected this disparity might be associated with the MCI diagnostic criteria used. Therefore, it was extremely urgent to unify the diagnostic criteria for MCI.

Like other organs, the brain will also undergo significant structural and functional changes (such as increased neuron loss, decreased synaptic density, declined energy production, disordered synaptic signaling and destructed protein homeostasis) with increasing age (Berchtold et al., 2008; Kohama et al., 2012). And cognitive functioning declines much faster among individuals with MCI than normal cognitive elderly (Cheng and Xiao, 2014). Inconsistent with many other studies (Li et al., 2013; Wang et al., 2017), we did not find that advanced age were a risk factor for MCI, and the difference might be due to the limited sample size.

Depression symptoms occur very commonly in people with MCI and the co-morbidity rate is about 44\% (Panza et al., 2010). Previous studies suggest that depression will promote the transformation of MCI into dementia (Barnes and Yaffe, 2011; Van der Mussele et al., 2014). In the present study, we also found that depression was a risk factor for developing MCI. There are several mechanisms that might be used to explain the above phenomenon, first, depression symptoms might increase the burden of amyloid in the cerebral cortex (especially precuneus, parietal, temporal, and occipital regions; Yasuno et al., 2016); second, depression symptoms might alter serotonin metabolism and reduce synaptic plasticity by increasing pro-inflammatory cytokines, such as interleukin-1 and tumor necrosis factor- $\alpha$ (TNF- $\alpha$; You et al., 2011); third, the concentration of BDNF in the brain of patients with depression is often significantly decreased, which might have a negative impact on the maintenance of neuronal homeostasis and modulation of synaptic plasticity (Lu et al., 2013). 
In addition, we also assessed the cognitive function of patients with diabetes. Compared with T2DM patients with normal cognitive function, T2DM patients with MCI showed a decline in both general and specific cognitive fields (Tables 3, 4). Previous study indicated that type 2 diabetes was associated with impairment of episodic memory and decreased executive function (including working memory, VF, processing speed, cognitive flexibility and cognitive control; McCrimmon et al., 2012; Yaffe et al., 2016), so our conclusions are consistent. By using partial correlation analysis (controlling for age, gender and education), we found that only the total score of AVLT was related to diabetes. And the results of linear regression suggested drug therapy (for diabetes) was associated with better cognitive performance $(t=2.263, p=0.025)$. However, we did not find any negative effect of diabetes on cognitive function in MCI patients. Since this was only a cross-sectional study, further research was needed on the relationship between T2DM and MCI.

There are several limitations to our study. First, this was only a cross-sectional study, and a causal relationship between cognition and T2DM could not be concluded. Second, we did not specifically discuss the relationship between the different subtypes of MCI and cognitive function. Third, longitudinal data from larger samples was needed to verify the above conclusions.

In summary, there is a high prevalence of MCI among Chinese T2DM patients. T2DM patients with MCI are impaired in multiple cognitive fields, but for patients with MCI, T2DM has no effect on cognitive function Depression is a risk factor for developing MCI, while education and use of glucose-lowering medications are protective ones.

\section{REFERENCES}

Arvanitakis, Z., Wilson, R. S., Bienias, J. L., Evans, D. A., and Bennett, D. A. (2004). Diabetes mellitus and risk of Alzheimer disease and decline in cognitive function. Arch. Neurol. 61, 661-666. doi: 10.1001/archneur.61.5.661

Barnes, D. E., and Yaffe, K. (2011). The projected effect of risk factor reduction on Alzheimer's disease prevalence. Lancet Neurology. 10, 819-828. doi: 10.1016/S1474-4422(11)70072-2

Berchtold, N. C., Cribbs, D. H., Coleman, P. D., Rogers, J., Head, E., Kim, R., et al. (2008). Gene expression changes in the course of normal brain aging are sexually dimorphic. Proc. Natl. Acad. Sci. U S A 105, 15605-15610. doi: 10.1073/pnas.0806883105

Cheng, Y., and Xiao, S. (2014). Recent research about mild cognitive impairment in China. Shanghai Arch. Psychiatry 26, 4-14. doi: 10.3969/j.issn.1002-0829.2014. 01.002

Cholerton, B., Baker, L. D., Montine, T. J., and Craft, S. (2016). Type 2 diabetes, cognition and dementia in older adults: toward a precision health approach. Diabetes Spectr. 29, 210-219. doi: 10.2337/ds16-0041

Cukierman, T., Gerstein, H. C., and Williamson, J. D. (2005). Cognitive decline and dementia in diabetes-systematic overview of prospective observational studies. Diabetologia 48, 2460-2469. doi: 10.1007/s00125-005-0023-4

Gao, Y., Xiao, Y., Miao, R., Zhao, J., Cui, M., Huang, G., et al. (2016). The prevalence of mild cognitive impairment with type 2 diabetes mellitus among elderly people in China: a cross-sectional study. Arch. Gerontol. Geriatr. 62, 138-142. doi: 10.1016/j.archger.2015.09.003

Gao, Y., Xiao, Y., Miao, R., Zhao, J., Zhang, W., Huang, G., et al. (2015). The characteristic of cognitive function in type 2 diabetes mellitus. Diabetes Res. Clin. Pract. 109, 299-305. doi: 10.1016/j.diabres.2015.05.019

Gil, L., Ruiz de Sánchez, C., Gil, F., Romero, S. J., and Pretelt Burgos, F. (2015). Validation of the montreal cognitive assessment (MoCA) in spanish as a

\section{DATA AVAILABILITY}

The raw data supporting the conclusions of this manuscript will be made available by the authors, without undue reservation, to any qualified researcher.

\section{ETHICS STATEMENT}

Ethical approval was obtained from Shanghai Mental Health Centre, and all the participants had signed an informed consent before the study was initiated.

\section{AUTHOR CONTRIBUTIONS}

WL wrote this article. LS analyzed the data. GL and SX were project leaders.

\section{FUNDING}

This work was supported by grants from the National Natural Science Foundation of China (number 81671402) and China Ministry of Science and Technology (2009BAI77B03).

\section{ACKNOWLEDGMENTS}

We extend our thanks to the main investigators from across the different regions of China: Juan Li, Wei Chen, Feng Bao, Yuping Wang, Huali Wang, Ying Liu, Xiaoyun Zuo, Zhongming Chen, Lijuan Cui and Mingyuan Zhang.

screening tool for mild cognitive impairment and mild dementia in patients over 65 years old in Bogota, Colombia. Int. J. Geriatr. Psychiatry 30, 655-662. doi: 10.1002/gps.4199

Gorska-Ciebiada, M., Saryusz-Wolska, M., Ciebiada, M., and Loba, J. (2014). Mild cognitive impairment and depressive symptoms in elderly patients with diabetes: prevalence, risk factors and comorbidity. J. Diabetes Res. 2014:179648. doi: 10.1155/2014/179648

Hong, X., Zhang, Z. X., Wu, L. Y., Shi, L. L., Zhao, X. H., and Wei, J. (2012). Validity of auditory verbal learning test in diagnosis of Alzheimer's disease. Zhongguo Yi Xue Ke Xue Yuan Xue Bao 34, 262-266. doi: 10.3881/j.issn.1000503X.2012.03.014

Kodl, C. T., and Seaquist, E. R. (2008). Cognitive dysfunction and diabetes mellitus. Endocr. Rev. 29, 494-511. doi: 10.1210/er.2007-0034

Kohama, S. G., Rosene, D. L., and Sherman, L. S. (2012). Age-related changes in human and non-human primate white matter: from myelination disturbances to cognitive decline. Age 34, 1093-1110. doi: 10.1007/s11357-0119357-7

Leung, J. L., Lee, G. T., Lam, Y. H., Chan, R. C., and Wu, J. Y. (2011). The use of the digit span test in screening for cognitive impairment in acute medical inpatients. Int. Psychogeriatr. 23, 1569-1574. doi: 10.1017/s1041610211000792

Li, X., Ma, C., Zhang, J., Liang, Y., Chen, Y., Chen, K., et al. (2013). Prevalence of and potential risk factors for mild cognitive impairment in community-dwelling residents of Beijing. J. Am. Geriatr. Soc. 61, 2111-2119. doi: 10.1111 /jgs. 12552

Li, W., Wang, T., and Xiao, S. (2016). Type 2 diabetes mellitus might be a risk factor for mild cognitive impairment progressing to Alzheimer's disease. Neuropsychiatr. Dis. Treat. 12, 2489-2495. doi: 10.2147/NDT.s111298

Lin, X., Haralambous, B., Pachana, N. A., Bryant, C., LoGiudice, D., Goh, A., et al. (2016). Screening for depression and anxiety among older Chinese immigrants living in Western countries: the use of the geriatric depression scale (GDS) 
and the geriatric anxiety inventory (GAI). Asia Pac. Psychiatry 8, 32-43. doi: 10.1111/appy.12191

Lu, B., Nagappan, G., Guan, X., Nathan, P. J., and Wren, P. (2013). BDNF-based synaptic repair as a disease-modifying strategy for neurodegenerative diseases. Nat. Rev. Neurosci. 14, 401-416. doi: 10.1038/nrn3505

Luchsinger, J. A., Reitz, C., Patel, B., Tang, M. X., Manly, J. J., and Mayeux, R. (2007). Relation of diabetes to mild cognitive impairment. Arch. Neurol. 64, 570-575. doi: 10.1001/archneur.64.4.570

McCrimmon, R. J., Ryan, C. M., and Frier, B. M. (2012). Diabetes and cognitive dysfunction. Lancet 379, 2291-2299. doi: 10.1016/S0140-6736(12)60360-2

O’Bryant, S. E., Humphreys, J. D., Smith, G. E., Ivnik, R. J., Graff-Radford, N. R., Petersen, R. C., et al. (2008). Detecting dementia with the mini-mental state examination in highly educated individuals. Arch. Neurol. 65, 963-967. doi: 10.1001/archneur.65.7.963

Panza, F., Frisardi, V., Capurso, C., D’Introno, A., Colacicco, A. M., Imbimbo, B. P., et al. (2010). Late-life depression, mild cognitive impairment and dementia: possible continuum? Am. J. Geriatr. Psychiatry 18, 98-116. doi: 10.1097/JGP.0b013e3181b0fa13

Petersen, R. C. (2011). Clinical practice. Mild cognitive impairment. N. Engl. J. Med. 364, 2227-2234. doi: 10.1056/NEJMcp0910237

Petersen, R. C., Smith, G. E., Waring, S. C., Ivnik, R. J., Tangalos, E. G., and Kokmen, E. (1999). Mild cognitive impairment: clinical characterization and outcome. Arch. Neurol. 56, 303-308. doi: 10.1001/archneur.56.3.303

Portet, F., Ousset, P. J., Visser, P. J., Frisoni, G. B., Nobili, F., Scheltens, P., et al. (2006). Mild cognitive impairment (MCI) in medical practice: a critical review of the concept and new diagnostic procedure. Report of the MCI working group of the european consortium on Alzheimer's disease. J. Neurol. Neurosurg. Psychiatry 77, 714-718. doi: 10.1136/jnnp.2005.085332

Smith, M. A., Zhu, X., Tabaton, M., Liu, G., McKeel, D. W. Jr., Cohen, M. L., et al. (2010). Increased iron and free radical generation in preclinical Alzheimer disease and mild cognitive impairment. J. Alzheimers Dis. 19, 363-372. doi: 10.3233/JAD-2010-1239

Van der Mussele, S., Fransen, E., Struyfs, H., Luyckx, J., Mariën, P., Saerens, J., et al. (2014). Depression in mild cognitive impairment is associated with progression to Alzheimer's disease: a longitudinal study. J. Alzheimers Dis. 42, 1239-1250. doi: 10.3233/JAD-140405

Wang, T., Xiao, S., Chen, K., Yang, C., Dong, S., Cheng, Y., et al. (2017). Prevalence, incidence, risk and protective factors of amnestic mild cognitive impairment in the elderly in Shanghai. Curr. Alzheimer Res. 14, 460-466. doi: $10.2174 / 1567205013666161122094208$
Xiao, S., Li, J., Tang, M., Chen, W., Bao, F., Wang, H., et al. (2013). Methodology of China's national study on the evaluation, early recognition and treatment of psychological problems in the elderly: the China longitudinal aging study (CLAS). Shanghai Arch. Psychiatry 25, 91-98. doi: 10.3969/j.issn.1002-0829. 2013.02.005

Xu, Z. P., Yang, S. L., Zhao, S., Zheng, C. H., Li, H. H., Zhang, Y., et al. (2016). Biomarkers for early diagnostic of mild cognitive impairment in type-2 diabetes patients: a multicentre, retrospective, nested case-control study. EBioMedicine 5, 105-113. doi: 10.1016/j.ebiom.2016.02.014

Yaffe, K., Peltz, C. B., Ewing, S. K., McCulloch, C. E., Cummings, S. R., Cauley, J. A., et al. (2016). Long-term cognitive trajectories and mortality in older women. J. Gerontol. A Biol. Sci. Med. Sci. 71, 1074-1080. doi: 10.1093/gerona/glw003

Yang, L., Shao, J., Bian, Y., Wu, H., Shi, L., Zeng, L., et al. (2016). Prevalence of type 2 diabetes mellitus among inland residents in China (2000-2014): a metaanalysis. J. Diabetes Investig. 7, 845-852. doi: 10.1111/jdi.12514

Yang, Q., Zhou, L., Liu, C., Liu, D., Zhang, Y., Li, C., et al. (2018). Brain iron deposition in type 2 diabetes mellitus with and without mild cognitive impairment-an in vivo susceptibility mapping study. Brain Imaging Behav. 12, 1479-1487. doi: 10.1007/s11682-017-9815-7

Yasuno, F., Kazui, H., Morita, N., Kajimoto, K., Ihara, M., Taguchi, A., et al. (2016). High amyloid- $\beta$ deposition related to depressive symptoms in older individuals with normal cognition: a pilot study. Int. J. Geriatr. Psychiatry 31, 920-928. doi: 10.1002/gps.4409

You, Z., Luo, C., Zhang, W., Chen, Y., He, J., Zhao, Q., et al. (2011). Pro- and anti-inflammatory cytokines expression in rat's brain and spleen exposed to chronic mild stress: involvement in depression. Behav. Brain Res. 225, 135-141. doi: 10.1016/j.bbr.2011.07.006

Conflict of Interest Statement: The authors declare that the research was conducted in the absence of any commercial or financial relationships that could be construed as a potential conflict of interest.

Copyright (C) $2019 \mathrm{Li}$, Sun, Li and Xiao. This is an open-access article distributed under the terms of the Creative Commons Attribution License (CC BY). The use, distribution or reproduction in other forums is permitted, provided the original author(s) and the copyright owner(s) are credited and that the original publication in this journal is cited, in accordance with accepted academic practice. No use, distribution or reproduction is permitted which does not comply with these terms. 